\title{
An approach to technological equipment performance information visualization system construction using augmented reality technology
}

\author{
Viktor Chekryzhov ${ }^{1, *}$, Ilya A. Kovalev ${ }^{1}$, and Anton S. Grigoriev ${ }^{1}$ \\ ${ }^{1}$ Moscow State University of Technology "STANKIN", 127055, Vadkovskii per. 1, Moscow, Russia
}

\begin{abstract}
The approach to obtaining the necessary information from heterogeneous technological equipment with its subsequent visualization is considered in the article. It is shown that the available interface screens of control systems are often overloaded with information, which makes it difficult to work with technological equipment. A variant of the solution of the terminal problem with application of the augmented reality technologies is proposed. The basic structural schemes and algorithms of operation of such a system are described. The first test version of the proposed solution is described.
\end{abstract}

To date, the technologies of augmented and virtual reality are developing at an active pace all over the world and already have a wide range of applications: education, healthcare, entertainment, design, real estate sales, advertising, etc. In addition, much attention is being paid to these technologies in the industrial sector, although it is still used more in test mode.

It is worth noting that in modern enterprises various solutions from different manufacturers (CNC systems, PLCs, etc.) are often used to manage various technological equipment and solve the tasks of automation of technological processes, which is caused by a variety of factors, including the economic component. This approach significantly complicates the linkage of all equipment with each other and the organization of a single information environment of the enterprise, which is currently an urgent task in the creation of digital and "smart" productions [1]. At the same time, attempts to introduce augmented reality (AR) technology mean tight binding to certain equipment and the impossibility of flexible work with equipment from different manufacturers with different data transfer protocols, etc.

At the same time, the complication of technological equipment and processes leads to a significant increase in the amount of information that must be obtained and analyzed in the course of the work, and, accordingly, to the increased requirements for the qualification of employees. At the same time, the tasks of realizing the human-machine interaction are complicated, on which the efficiency of production as a whole and the safety at work depends [2-3].

* Corresponding author: vchekryzhov@yandex.ru 
At the current stage of development, more attention is paid to the introduction of smart devices, such as tablet computers, mobile phones, into production processes. However, there are certain problems associated with the lack of ways to present large amounts of information, their systematization, as well as approaches that provide the operator with only the information that is most important and necessary at the moment [4-5]. In other words, there is a problem of redundancy of the information transferred to the operator: overloaded interfaces of the terminal part, each control system has its own technological screens, with different arrangement of windows for setting system parameters, selecting control programs, binding of machine axes, referencing the system, etc.

Another important task can be considered the problem of maintaining complex technological equipment, and training personnel to work with it. This is usually decided by the organization of specialized courses and the involvement of outside specialists, which also entail additional financial and time costs [6].

One of the solutions to the problems described can be the introduction of augmented reality technology on production, which, on the one hand, will significantly increase the information content of the ongoing technological processes, and on the other, reduce the amount of redundant information that the operator does not need at a certain moment. At the same time, visualization of technological processes will be as clear as possible, and the level of dangerous situations caused by inexperience of the operator will be minimized. In training, this approach will allow the learner to focus on key and meaningful parameters, and also free from the need to study a complex interface [7]. By running the simulation mode, the learner can observe the virtual processing process, monitor the machine parameters and perform exercises simulating emergency situations without the risk of damaging the process equipment and injuring yourself.

Such an approach can be realized with the help of various devices, among which one can distinguish portable devices (mobile devices, tablet computers), stationary and projection systems, glasses and lenses of augmented reality.

\begin{tabular}{|c|c|c|c|}
\hline \multicolumn{4}{|c|}{$\begin{array}{c}\text { Display devices } \\
\text { (mobiles, tables, smartglasses) }\end{array}$} \\
\hline $\begin{array}{l}\text { Android AR } \\
\text { SDK }\end{array}$ & \multicolumn{2}{|c|}{ IOS AR SDK } & HTTP request \\
\hline \multicolumn{4}{|c|}{ AR Server } \\
\hline \multicolumn{4}{|c|}{\begin{tabular}{|c|} 
Data Processing Server \\
Database (command and current values)
\end{tabular}} \\
\hline \multicolumn{4}{|c|}{$\begin{array}{l}\text { Communication layer } \\
\text { Various digital interfaces, fieldbus } \\
\text { (RS435,CANOpen,Modbus, etc.) }\end{array}$} \\
\hline \multicolumn{2}{|c|}{$\begin{array}{c}\text { I/O control } \\
\text { Expansion cards, drivers }\end{array}$} & \multicolumn{2}{|c|}{ OPC UA, SCADA } \\
\hline \multicolumn{4}{|c|}{$\begin{array}{l}\text { Technological equipment } \\
\text { Machine tools of various manufacturers, } \\
\text { electrical cabinets, industrial robots }\end{array}$} \\
\hline
\end{tabular}

Fig. 1. Architectural model of the proposed solution 
Figure 1 shows the architectural model of the proposed solution. This model consists of several levels.

The lower level is a heterogeneous technological equipment of various manufacturers. It can be either simple lathes or complex five-axis machining centers with manipulators for feeding blanks to the processing zone.

The next level is technologies and standards that allow you to collect information from equipment. It can be standard solutions (OPC UA) or well as proprietary solutions that support interfaces for the exchange of data between technological devices (EtherCAT, Sercos III, etc.) [8].

The communication layer contains modules of various digital interfaces for communicating with the lower layers and transmitting information to the AR layer.

There are ready-made solutions built on OPC technology that can be integrated into the target management system. Creating their own systems for data collection and processing has certain advantages: flexibility of customization for own system, simplicity of modernization, integration into the management system at the level of communication protocols, etc.

The communications layer of the component contains various digital interfaces for communicating with the lower layers and transmitting information to the AR layer. AR server is a web application or a stationary client that performs the tasks:

- Processes requests from the client application;

- Gets information about the state of the process equipment from the OPC UA component / own solutions (I/O control);

- Processes the received data, prepares the answer;

- Sends a response to the client.

The server component contains of a subcomponent for implementing visualization models, which verifies and prepares the necessary drawing paths, and interacts with AR devices. To transfer to the upper layer, requests are used for the developed protocol over the existing HTTP.

The upper level is a special device for working with AR (tablets, glasses, etc.) with a special SDK.

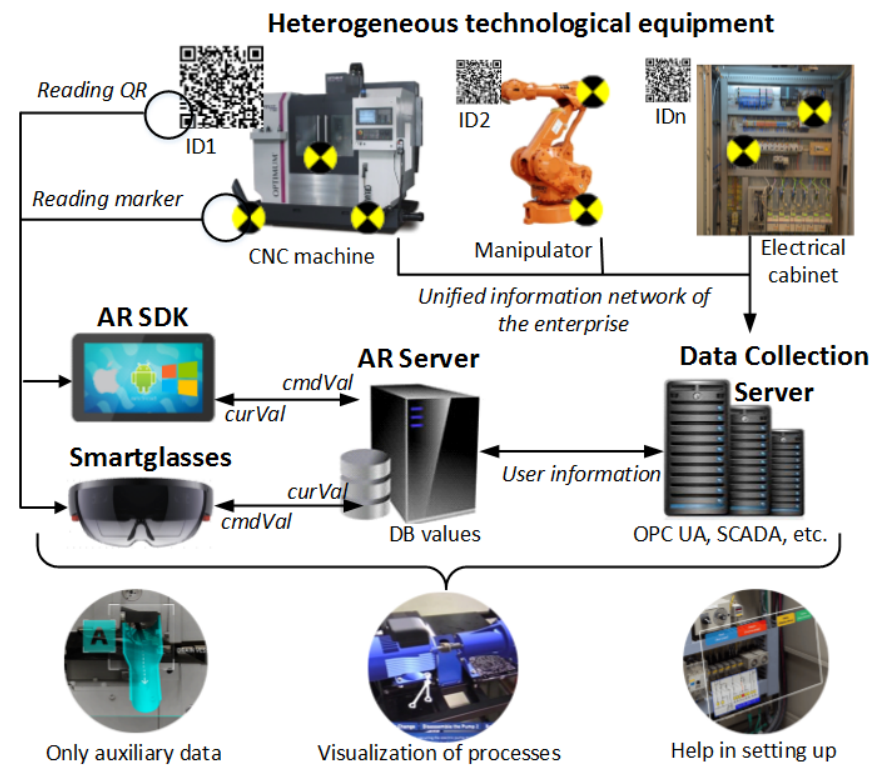

Fig. 2. Structural diagram of the proposed solution 
Figure 2 shows the block diagram of the proposed solution. This diagram shows the elements of the system and shows their interactions. Each technological equipment is assigned a unique ID, which is entered in the database on the server. The process equipment is connected to the OPC UA server by industrial protocols, standard EtherNet or other protocols supported by the equipment. The connection between the information processing server and the data collection server is carried out by means of TCP / IP [9]. In general, the data collection server and the information processing north can be the same device when replacing the OPC UA server with its own solution, as shown above.

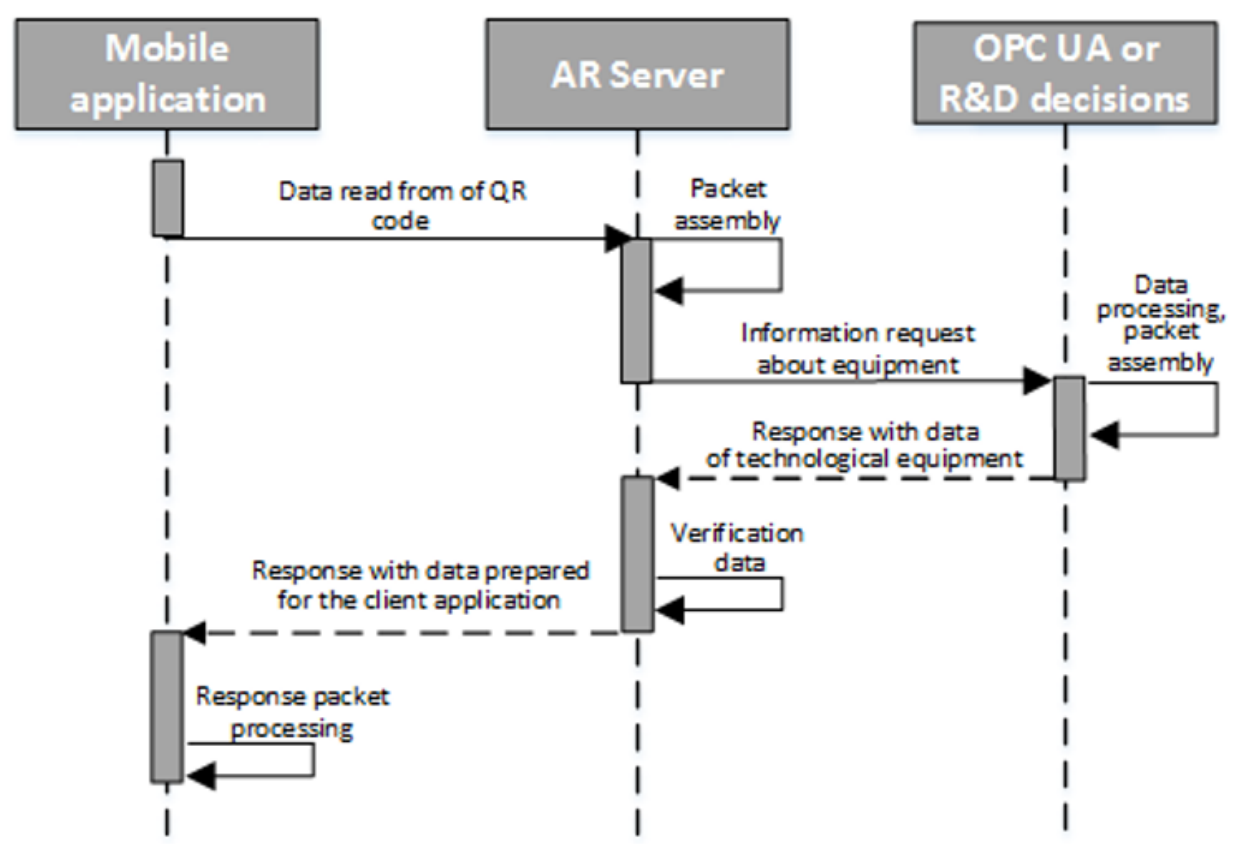

Fig. 3. Sequence diagram

Figure 3 shows a simplified diagram of the sequence of the proposed solution. It starts with the AR device reads the marker using the built-in camera. The client application is waiting for the marker to appear in the camera area. After receiving the token, a request is sent from the server, it in turn sends the request to the OPC UA (or I/O control) and requests information about the equipment. Then the client receives a response from the server displays the information on the screen if the marker is still in the scope of the camera, then the application again sends the request, as long as the marker is in visibility. User chooses the necessary data himself when configuring the system. 

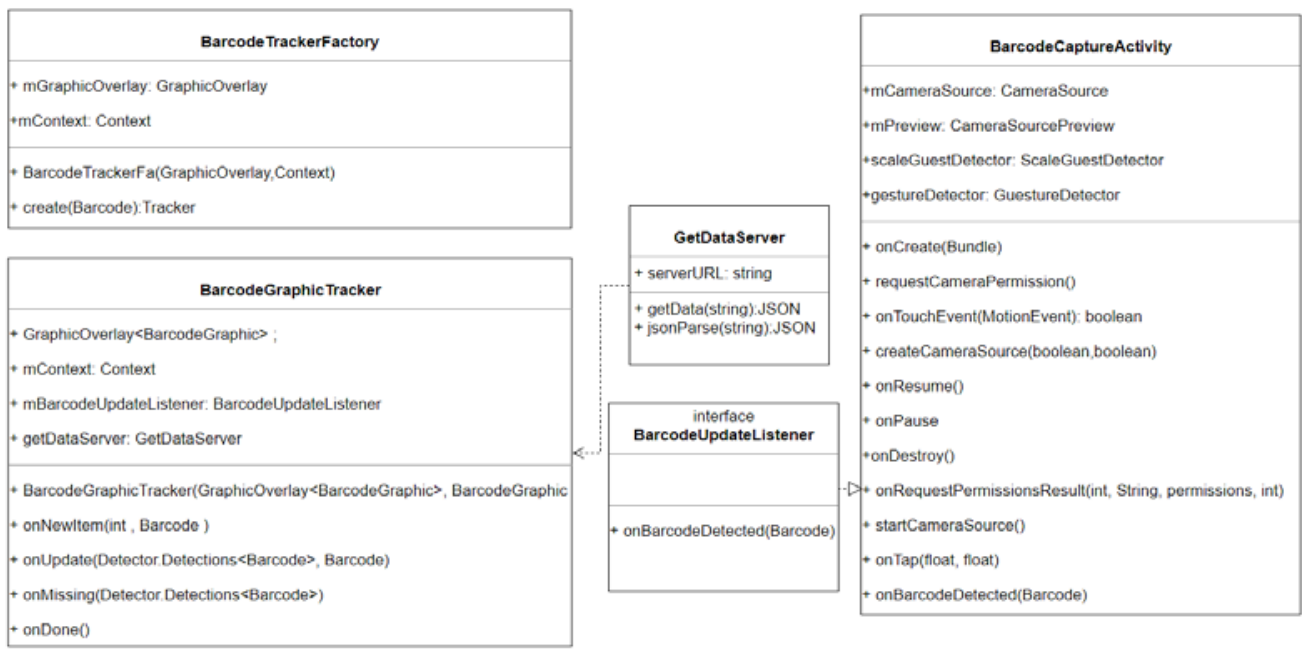

Fig. 4. Class diagram of the client application

Figure 4 shows a fragment of the class diagram of the the proposed solution, the main fields of which are:

- BarcodeGraphicFactory - Factory for creating TrackerFactory objects;

- BarcodeGraphicTracker - An object of this class monitors the position of the QR code on the application screen;

- GetDataServer - Class, for requests to the server with data;

- BarcodeUpdateListener - Interface, implemented in BarcodeCaptureActivity;

- BarcodeCaptureActivity - The application window on which it occurs, capture the QR code and draw information about the process equipment.

The first results were tested using a 4-axis milling machine under the control of the national control system "AxiOMA Control", developed at MSTU "Stankin".

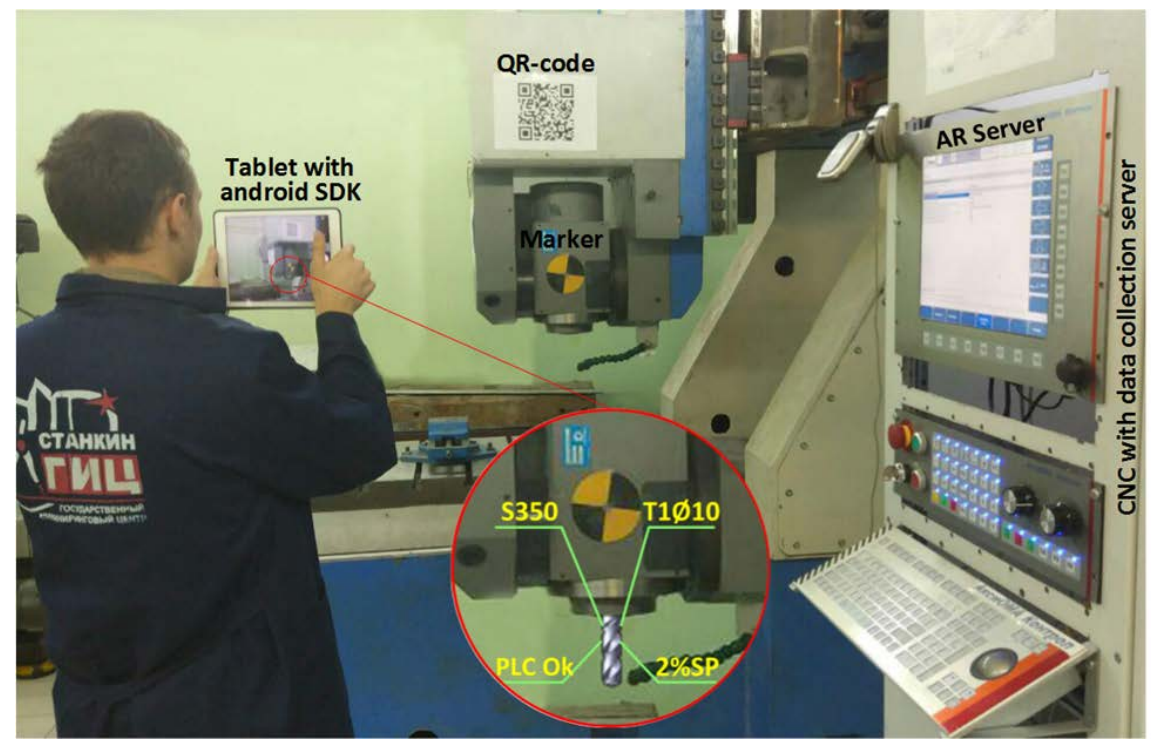

Fig. 5. Testing 4-axis milling with CNC system "AxiOMA Control" and AR server 
This CNC system has an open modular architecture that allows you to add new innovative solutions without losing the previous functionality [10-11].

AR server was successfully installed (in test mode) on a real-time machine with LinuxRT. In the terminal of the CNC system "AxiOMA Control", a special program module for communicating with the AR server was added [12]. The first test tests using a tablet with the SDK installed showed the correctness of the approach used - on the screen of the tablet could display only the information that is needed (PLC state, number of tool, etc.) at a given moment. Also, track the movements of the instrument using its AR component.

To visualize the processed data, the client component was used, which is installed on smart tablet devices, smartphones running the android operating system. In the application using the SDK implemented technology AR.

The server was implemented using the java language using servlet technology. Thus, the server can be deployed on a Windows or Linux platform, which allows you to choose the optimal solution depending on the tasks [13-14]. This approach will allow you to be not attached to any one execution platform.

The connection between the client application and the information processing north is implemented using the HTTP protocol. In this case, the AR devices and the informationprocessing server are constantly exchanging JSON files containing the information necessary to the user.

As the development of the project, in addition to developing models and algorithms for aggregating information with subsequent large-scale visualization, a method will be developed for porting the server with a database to a single-board computer [15]. This will allow it to be connected to the network at once from several technological units or to a separate OPC UA server, while portability the device will not be tied to any one machine or even a shop, which is an undoubted advantage in the testing stages.

This research was supported by the Ministry of Education and Science of the Russian Federation as a public program in the sphere of scientific activity (N 2.1237.2017/4.6).

\section{References}

1. Nikishechkin, P., Kovalev, I. and Nikich, A. (2017). An approach to building a crossplatform system for the collection and processing of diagnostic information about working technological equipment for industrial enterprises. MATEC Web of Conferences, 129, p.03012.

2. Martinov, G., Kozak, N. and Nezhmetdinov, R. (2017). Implementation of Control for Peripheral Machine Equipment Based on the External Soft PLC Integrated with CNC. International Conference on Industrial Engineering, Applications and Manufacturing (ICIEAM), pp.1-4.

3. R. Neugebauer, B. Denkena, K. Wegener, "Mechatronic Systems for Machine Tools," in CIRP ANNALS-MANUFACTURING TECHNOLOGY, vol. 56, no. 2, pp. 657-686, 2007.

4. Martinov, G., Kozak, N., Nezhmetdinov, R., Grigoriev, A., Obukhov, A. and Martinova, L. (2017). Method of decomposition and synthesis of the custom CNC systems. Automation and Remote Control, 78(3), pp.525-536.

5. Martinov, G., Sokolov, S., Martinova, L., Grigoryev, A. and Nikishechkin, P. (2017). Approach to the Diagnosis and Configuration of Servo Drives in Heterogeneous Machine Control Systems. In: 8th International Conference, ICSI. Fukuoka, Japan, pp.586-594. 
6. G.M. Mrtinov, N.V. Kozak, R.A. Abdullaev, I.A. Kovalev. Construction of a specialized distributed control system for a precision machining center VMG 50. Automation in the industry.№6. P. 16-20. (2014)

7. Martinov, G., Obuhov, A., Martinova, L. and Grigoriev, A. (2014). An Approach to Building Specialized CNC Systems for Non-traditional Processes. Procedia CIRP, 14, pp.511-516.

8. Kovalev, I., Nikishechkin, P., and Grigoriev A. (2017) Approach to Programmable Controller Building by its Main Modules Synthesizing Based on Requirements Specification for Industrial Automation. International Conference on Industrial Engineering, Applications and Manufacturing (ICIEAM), pp.1-4

9. Martinov, G., Lyubimov, A., Bondarenko, A., Sorokoumov, A. and Kovalev, I. (2015). An approach to building a multiprotocol CNC system. Automation and Remote Control, 76(1), pp.172-178.

10. I.A Kovalev, S.V. Sokolov, L.I. Martinova, S.V. Rybnikov. Construction of a specialized CNC system for thread grinding machines. Automation in the industry. №5. P.38-40. (2015)

11. Martinov, G., Obukhov, A. and Kozak, N. (2018). The Usage of Error Compensation Tools of CNC for Vertical Milling Machines. Russian Engineering Research, 38(2), pp.119-122.

12. Martinov, G. and Kozak, N. (2016). Specialized numerical control system for five-axis planing and milling center. Russian Engineering Research, 36(3), pp.218-222.

13. M.F. Cloutier, C. Paradis, V.M. Weaver, "Design and analysis of a 32-bit embedded high-performance cluster optimized for energy and performance," in Hardware-Software Co-Design for High Performance Computing (Co-HPC) IEEE, pp.1-8, 2014.

14. Jian-Yu Chen, Kuo-Cheng Tai, Guo-Chin Chen, "Application of Programmable Logic Controller to Build-up an Intelligent Industry 4.0 Platform" in Proc. CIRP, vol. 63, pp. $150-155,2017$

15. Grigoriev, S. and Martinov, G. (2016). An ARM-based Multi-channel CNC Solution for Multi-tasking Turning and Milling Machines. Procedia CIRP, 46, pp.525-528. 